\title{
Green Deal - Chancen und Risiken für die Mineralrohstoffwirtschaft
}

\author{
Roman Stiftner \\ Fachverbände Bergbau-Stahl und NE-Metallindustrie, Wirtschaftskammer Österreich, Wien, Österreich
}

Eingegangen 1. Oktober 2020; angenommen 6. Oktober 2020; online publiziert 28. Oktober 2020

\begin{abstract}
Zusammenfassung: Covid-19 hat die Mineralrohstoffwirtschaft stark getroffen. Produktionsrückgänge und Nachfrageeinbrüche prägen das Jahr 2020. Die Verschärfung des EU-Klimaziels (Green Deal) für 2030 von 40 auf $55 \%$ seitens der EU-Kommission stellt die gesamte Europäische Industrie, nicht zuletzt die energieintensive, zusätzlich vor große Herausforderungen. Die Belastungen für einzelne Branchen und den Industriestandort müssen unbedingt bedacht werden, möchte man ein Carbon-Leakage-Szenario vermeiden und Europa trotz ambitionierter Klimapolitik wettbewerbsfähig halten. Das europäische Vorhaben einer Grenzsteuer (Carbon Border Tax Adjustment) und nachhaltige Finanzierungsformen (Sustainable Finance und Taxonomy) werden widersprüchlich diskutiert. Es gilt, nicht nur das Klima, sondern zugleich die Europäische Industrie, Arbeitsplätze sowie das Sozialsystem zu schützen. Die Transformation zu einer nachhaltigen Wirtschaft und Gesellschaft erfordert einen großen Mehrbedarf an mineralischen Rohstoffen und elektrischer Energie. Nur durch eine Stärkung der Wertschöpfungsketten kann eine ausreichende Rohstoffversorgung gewährleistet werden.
\end{abstract}

Schlüsselwörter: Green Deal, Kreislaufwirtschaft, Mineralrohstoffwirtschaft, Carbon-Leakage,

Grenzsteuerausgleichsmechanismus

Green Deal-Chances and Risks for the Mineral Raw Materials Industry

Abstract: The Mineral Raw Materials Industry was hit severely by COVID-19. Declines in production and collapsing demands characterize the year 2020. The EU

$\overline{\text { Dipl.-Ing. R. Stiftner ( } \triangle)}$

Fachverbände Bergbau-Stahl und NE-Metallindustrie,

Wirtschaftskammer Österreich,

Wiedner Hauptstr. 63,

1045 Wien, Österreich

roman.stiftner@wko.at
Commission's proposal to raise the 2030 greenhouse gas emission reduction targets, including emissions and removals from 40 to at least $55 \%$, poses a challenge for the European industry, especially the energy-intensive one. The European Commission's idea for a carbon border tax adjustment mechanism and sustainable finance models (sustainable finance and taxonomy) are currently the subject of controversial debate. Economic burdens on specific branches and the industrial site have to be taken into account, if a carbon leakage scenario is to be avoided and if Europe has to be given a fair chance to stay competitive in spite of an ambitious climate policy. It is important, not only to protect climate and environment, but also the European industry, jobs and social system. The transformation towards a sustainable economy and society necessitates an increasing demand of mineral raw materials and electrical energy. Only through developing resilient value chains a sufficient supply of raw materials can be achieved.

Keywords: Green Deal, Circular economy, Mineral raw materials, Carbon-leakage, Carbon border tax adjustment

\section{COVID-19-Pandemie und ihre Auswirkun- gen auf die Mineralrohstoffwirtschaft}

Die Mineralrohstoffwirtschaft ist aufgrund der Nachfragerückgänge durch COVID-19, Handelsspannungen, protektionistische Maßnahmen, ungleiche Wettbewerbsbedingungen sowie umweltpolitische Herausforderungen in der Krise besonders gefordert.

Der österreichische Bergbau und Stahl-Sektor sowie die Nichteisenmetall-Industrie (NE-Industrie) leiden vor allem unter dem Rückgang der Automobil- und Luftfahrt- 
Abb. 1: Globale Rohstahlproduktion, August 2020. (https://doi.org/https://www. worldsteel.org/media-centre/ press-releases/2020/August2020-crude-steel-production. html [28.09.2020])
Abb. 2: Änderungen der Außenhandelsbilanz: Zeitraum 2013-2019 (in Mio.t, fertige Stahlerzeugnisse). (EUROFER: THE EUROPEAN STEEL ASSOCIATON: "Situation of the European Steel Industry, $21 \mathrm{Sep}$ tember 2020“ [28.09.2020])

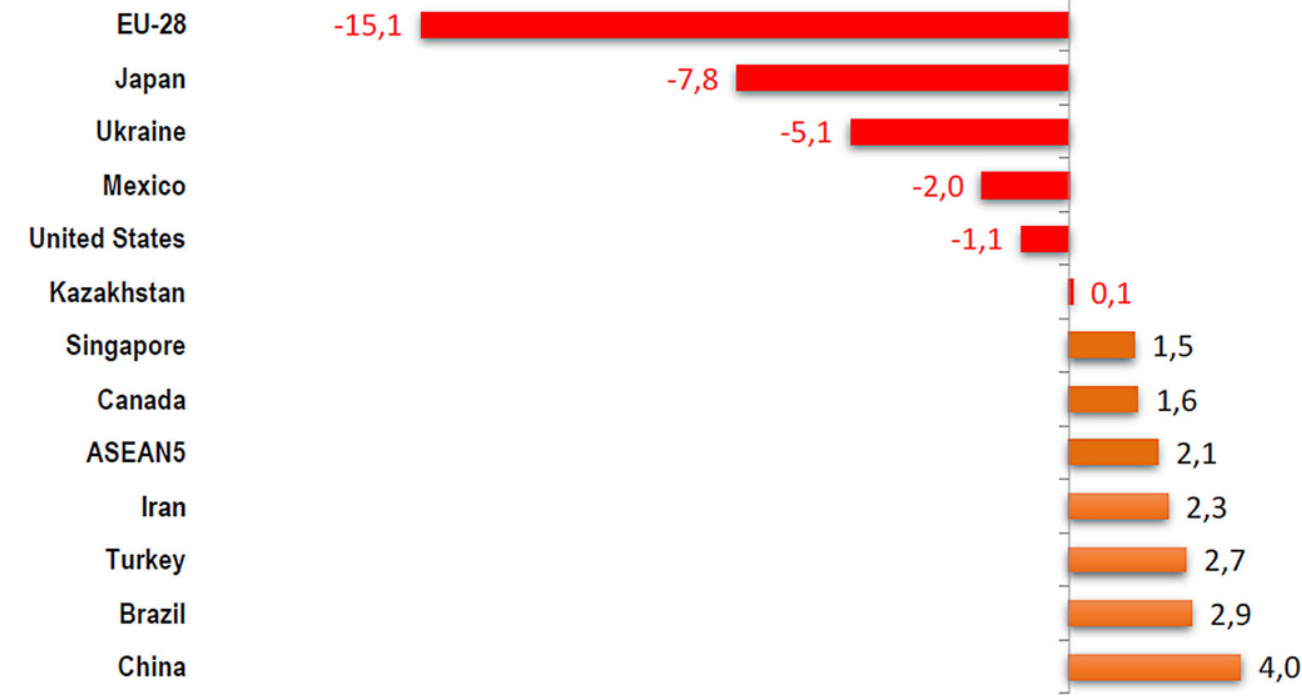

branche ${ }^{1,2,3}$, aber auch an der akuten, generellen Wirtschaftsflaute im In- und Ausland. ${ }^{4}$ So ist derzeit in Österreich bedingt durch die Corona-Krise für 2020 von einem BIP-Verlust von $-35,7 \mathrm{Mrd}$ € gegenüber dem Vorjahr auszugehen. ${ }^{5}$ Der Shutdown der Europäischen Stahlindustrie hat zu einem Nachfragerückgang von rund $50 \%$ zwischen Mitte März und Mai geführt. Stahlunternehmen mussten im Vergleich zum Vorjahr Produktionsrückgän-

\footnotetext{
1 https://industriemagazin.at/a/lufthansa-kuendigungen-und-150flugzeuge-weniger [25.09.2020].

2 https://industriemagazin.at/a/aua-chef-erwartet-in-der-luftfahrtweitere-einschnitte[25.09.2020].

3 https://www.automotive.at/kfz-wirtschaft/das-kostet-die-coronakrise-201041 [25.09.2020].

4 https://www.wto.org/english/news_e/news20_e/stat_23sep20_e.htm [28.09.2020].

5 https://de.statista.com/statistik/daten/studie/1117995/umfrage/ wirtschaftliche-verluste-infolge-der-corona-krise-in-oesterreich/ [28.09.2020].
}

ge von durchschnittlich $23 \%$ verzeichnen (Stand August 2020). ${ }^{6}$

Erschwerend wiegt dabei der Umstand, dass sich China augenscheinlich von den negativen Covid-19-Auswirkungen schneller zu erholen scheint als Europa. So ist laut einer im September 2020 erschienen Auswertung der World Steel Association die Produktion von Roheisen in China im August 2020 verglichen mit dem Vorjahr sogar um 8,4\% gestiegen (s. Abb. 1). Dagegen ist die Stahlproduktion in Deutschland um 13,4\% gefallen und Spanien verzeichnet Produktionsrückgänge von über 32\%. 2019 produzierte China mit 996,3 Mio.t rund neunmal so viel Rohstahl wie Indien. Nur etwa 5\% der gesamten chinesischen Rohstahlproduktion wurden 2019 exportiert. Dennoch ist das Land mit Nettoexporten in Höhe von 48,3 Mio.t der größte Nettoexporteur von Rohstahl der Welt, vor Japan (26,7 Mio.t),

\footnotetext{
6 EUROFER: THE EUROPEAN STEEL ASSOCIATON: "Situation of the European Steel Industry, 21 September 2020" [28.09.2020].

7 https://www.worldsteel.org/media-centre/press-releases/2020/ August-2020-crude-steel-production.html [25.09.2020].
} 


\section{DER ANTEIL DER EU AN DEN \\ KLIMASCHÄDLICHEN EMISSIONEN SINKT VON 16\% AUF $6 \%$ \\ (ZEITRAUM 1990 - 2030)}

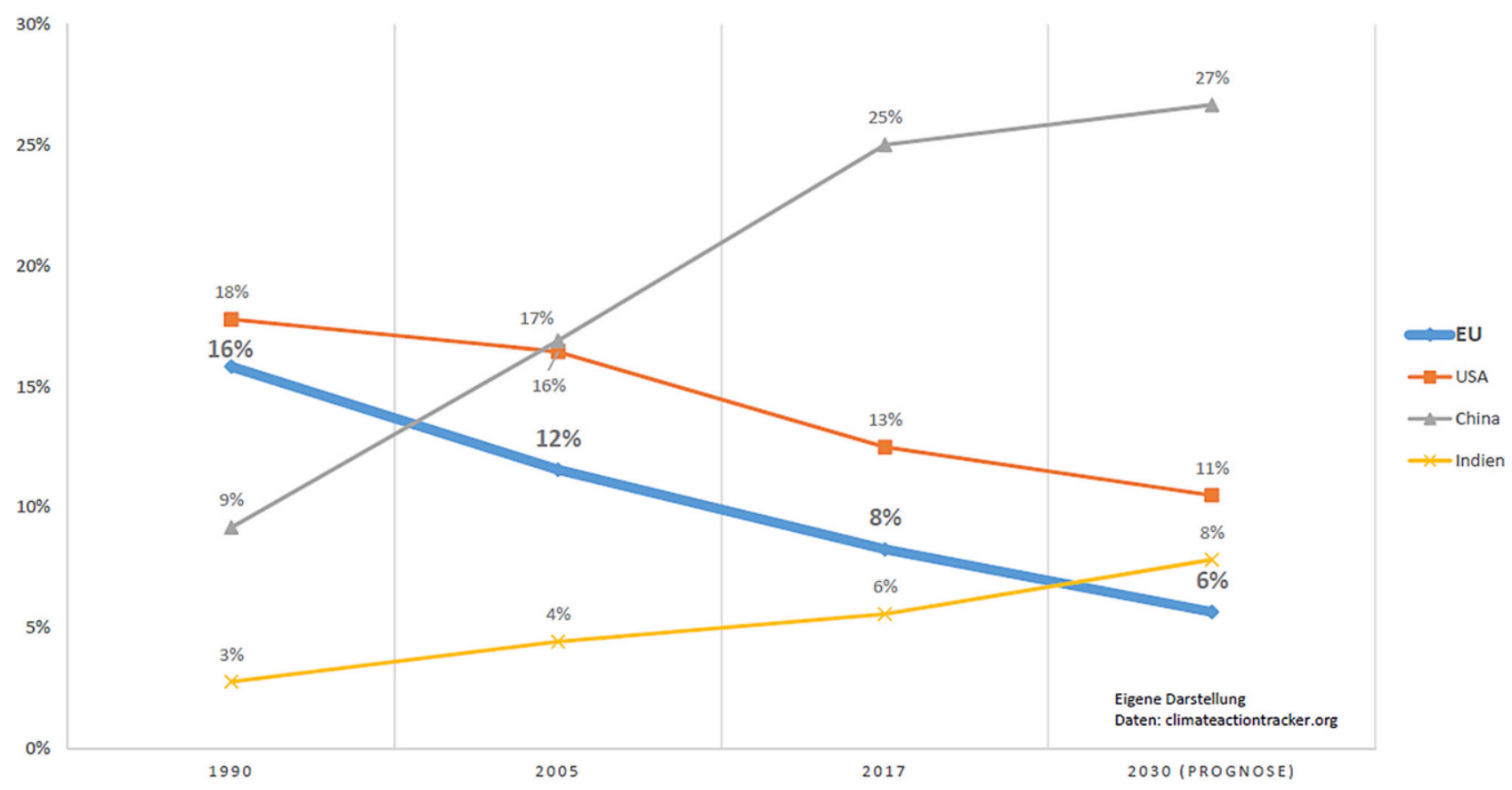

Abb. 3: KlimaschädlicheEmissionen: Anteil derEU sinkt in 2030 auf $6 \%$. (https://news.wko.at/news/oesterreich/bsi_up_klimaschaedliche_emissionen_ anteil_eu.pdf [25.09.2020])

Russland (22,7 Mio.t) und der Ukraine (14,0 Mio.t). ${ }^{8}$ Das steigende EU-Handelsbilanzdefizit im Bereich der fertigen Stahlerzeugnisse von 15,1 Mio.t verglichen mit einem Plus von 4 Mio. auf Seiten Chinas zeigt weiters dringenden Handlungsbedarf (s. Abb. 2).

\section{Klima- und Wirtschaftspolitik: Suche nach der richtigen Balance}

Österreich hat in gemeinsamer Linie mit der EU ambitionierte Klimaziele festgelegt, um weltweit zu einer Reduktion von $\mathrm{CO}_{2}$-Emissionen beizutragen. Die hohen Umweltstandards in Österreich bergen dabei allerdings die Gefahr der Auslagerung der Produktion ins Ausland, das über weniger strenge Standards verfügt. Bedenkt man, dass der Anteil der EU an globalen THG-Emissionen bis zum Jahr 2030 weniger als $6 \%$ (s. Abb. 3) betragen wird, wird außerdem deutlich, dass für die Erreichung des globalen 1,5Grad-Ziels die Treibhausgasemissionen in China und den USA (s. Abb. 4) den wesentlichen Faktor spielen.

Österreich hat im Vergleich zu anderen Herstellländern, aus welchen derzeit Produkte importiert werden, einen hohen technologischen Standard und niedrige Emissionsintensität. Eine verstärkte Produktion in Österreich würde einen wesentlichen Beitrag zum globalen Klimaschutz

\footnotetext{
8 https://deutsche-wirtschafts-nachrichten.de/504569/China-bautweltweit-fuehrende-Stellung-beim-Stahl-aus Verfasst: 14.06.2020 [28.09.2020].
}

leisten. So führt nach einer Studie der österreichischen Energieagentur und des Austrian Institute of Technology (climApro, 2019) ${ }^{9}$ eine zusätzliche nationale Produktion zwar auch zu Mehremissionen in Österreich, dem stehen aber - global gesehen - in der gesamten Wertschöpfungskette deutlich größere Einsparungen gegenüber. Umgekehrt würden als Folge einer Produktionsverlagerung ins Ausland inklusive Transportkosten die Emissionen um 1,9 t steigen, während in Österreich eine Tonne $\mathrm{CO}_{2}$ weniger emittiert werden würde. ${ }^{10}$ Eine Abwanderung der energieintensiven Industrie hätte daher nicht nur den Verlust von Beschäftigung und Know-how in Österreich und Europa zur Folge, es würde auch die Klimabilanz im Verhältnis 1:2 verschlechtern und uns von den Paris-Zielen weiter entfernen (s. Abb. 5).

Die derzeitige Carbon-Leakage-Regelung sieht folgendes vor: „Zum Erhalt der Wettbewerbsfähigkeit von unter das EU-EHS fallenden Branchen erhalten Sektoren und Teilsektoren, bei denen davon ausgegangen wird, dass sie einem erheblichen Carbon-Leakage-Risiko ausgesetzt sind, in Phase 3 des EU-EHS (2013-2020) gegenüber anderen Industrieanlagen einen höheren Anteil kostenloser

\footnotetext{
9 https://www.wko.at/branchen/industrie/climapro-studie-industrielleoekologie.html[28.09.2020].

10 Institut für Industrielle Ökologie: Potenzialanalyse von veränderten Produktionsstrukturen in der österreichischen Industrie für globalen Klimaschutz und ihre monetären Auswirkungen (2019): https://www.wko. at/branchen/industrie/climapro-studie.pdf[18.09.2020].
} 


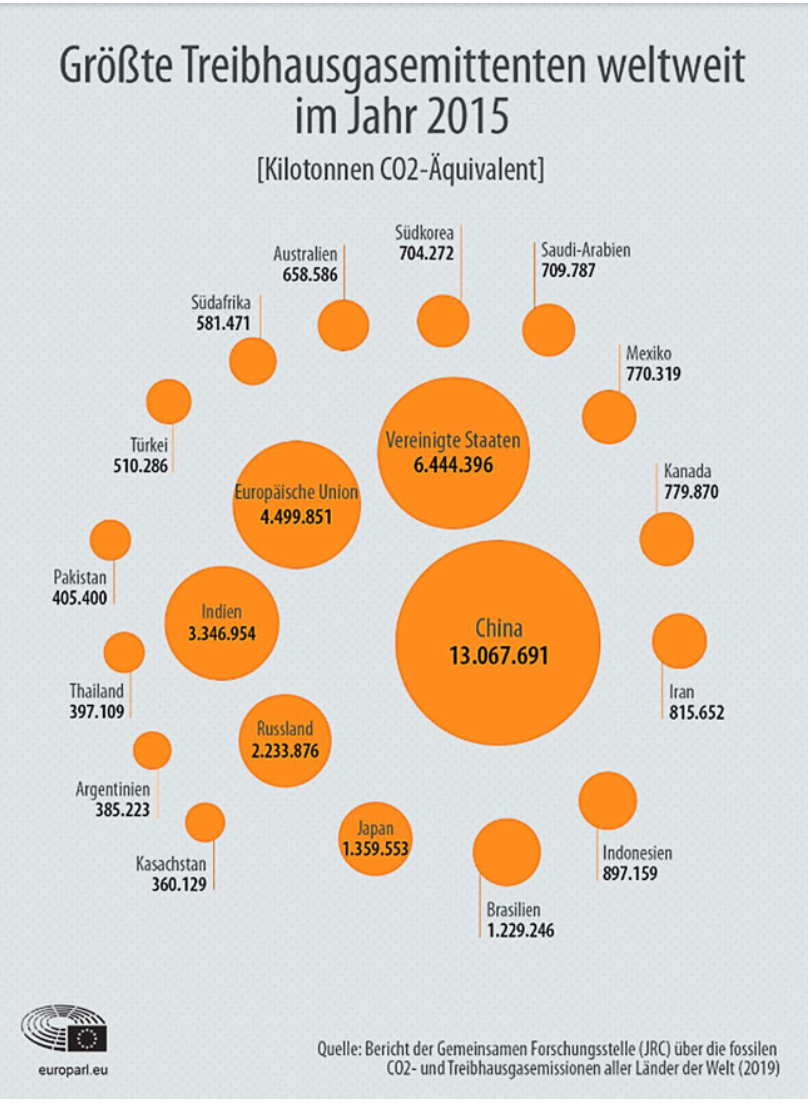

Abb. 4: Die größten Treibhausgasemittenten weltweit im Jahr 2015. (https://doi.org/https://www.europarl.europa.eu/news/de/headlines/ society/20180301STO98928/treibhausgasemissionen-nach-landernund-sektoren-infografik [25.09.2020])

Zertifikate.“ ${ }^{11}$ Durch die Verschärfung der europäischen Klimaziele wurde die kostenlose Zuteilung von Emissionszertifikaten an Carbon-Leakage gefährdete Sektoren laufend reduziert. ${ }^{12}$ Betriebe müssen seither vermehrt Zertifikate zukaufen.

Der Green Deal ${ }^{13}$ der Europäischen Kommission wird nicht ohne fairen Wettbewerb im Handel und ausgeglichene Umweltkosten umsetzbar sein (s. Abb. 6). Der Mineralrohstoffsektor und hier insbesondere der Stahlbereich haben den größten Hebeleffekt auf dem Weg zu einem klimaneutralen Europa. Unerlässlich ist es, dass die europäischen industriellen Wertschöpfungsketten intakt bleiben. Es muss in Europa gelingen, durch treibhausgasarme Prozesse Wettbewerbsvorteile zu erzielen und $\mathrm{CO}_{2}$-arme Technologien als Innovationsführer auch außerhalb der EU zu exportieren. Umso entscheidender ist es, Rahmen- und Förderbedingungen für eine erfolgreiche Transformation der Europäischen Industrie zu schaffen.

Ziel muss es daher sein, ein internationales „Level Playing Field“ sicherzustellen. Dies kann auf Dauer nur durch

\footnotetext{
11 https://ec.europa.eu/clima/policies/ets/allowances/leakage_de [25.09.2020].

12 https://ec.europa.eu/clima/policies/ets/allowances_de [25.09.2020].

$13 \mathrm{https} / /$ ec.europa.eu/info/strategy/priorities-2019-2024/europeangreen-deal_de[25.09.2020].
}

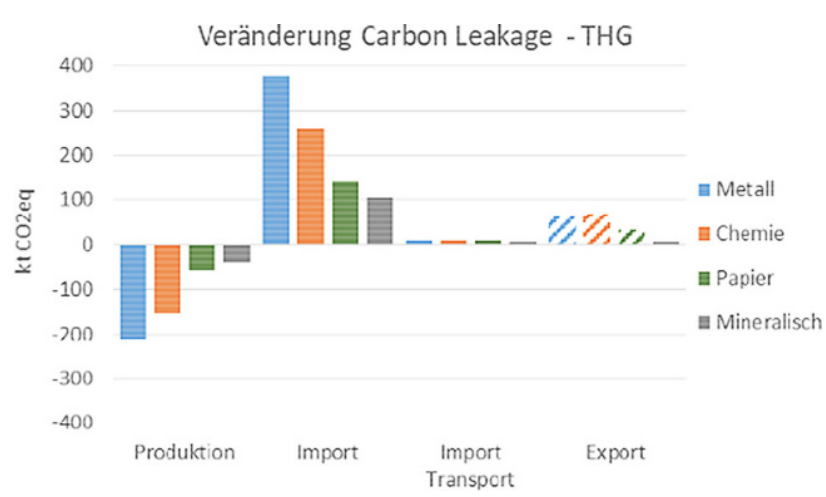

Abb. 5: Veränderungen der THG-Emissionen im "Carbon-Leakage"Szenario (https://www.wko.at/branchen/industrie/climapro-studie.pdf [18.09.2020].)

einen international einheitlichen $\mathrm{CO}_{2}$-Preis oder einen effektiven WTO-konformen Umweltzoll erreicht werden (s. Abschn. 6).

Die österreichische Metall- und Bergbauindustrie forscht unterdessen intensiv an Break-Through-Technologien, die die Erfüllung der europäischen Klimaziele ermöglichen sollen. Beispielsweise wird untersucht, wie Stahl im industriellen Maßstab nahezu treibhausgasneutral hergestellt werden kann. Fossile Stoffe werden hierbei durch Wasserstoff und elektrische Energie ersetzt. Eine besondere Herausforderung liegt in der Bereitstellung hoher Mengen nachhaltig erzeugter, elektrischer Energie.

Für die wasserstoffbasierte Stahlherstellung in Österreich würde zusätzlich die enorme Menge von ca. 25TWh elektrische Energie gebraucht. Für die klimaneutrale Transformation der österreichischen Industrie würde mehr als das Doppelte des derzeit in Österreich verbrauchten elektrischen Stroms benötigt. ${ }^{14}$

Kernfragen auf den Weg der Transformation hin zu einer treibhausgasneutralen Gesellschaft in Österreich und Europa sind daher:

- Verfügen wir rechtzeitig über benötigte Break-ThroughTechnologien?

- Sind diese $\mathrm{CO}_{2}$-neutralen Prozesse am globalen Markt wettbewerbsfähig und werden die Zusatzkosten für Errichtung und Betrieb staatlich ausgeglichen?

- Kann der stark ansteigende Mehrbedarf an mineralischen und metallischen Rohstoffen gedeckt werden?

- Ist der hohe Bedarf an zusätzlicher erneuerbarer elektrischer Energie, die letztendlich fossile Brennstoffe ersetzen soll, zu wettbewerbsfähigen Kosten vorhanden?

\footnotetext{
14 Austrian Energy Agency, Austrian Institute of Technology: Im Wettbewerbum dieZukunft. Klimapolitische Perspektiven für den Beitrag derösterreichischen Industrie zur Treibhausgasneutralität (2019): https:// www.wko.at/branchen/industrie/zukunft-policy-paper.pdf[18.09.2020].
} 
Abb. 6: Der Betrag der Stahlindustrie zu Erreichung der Klimaziele des Green Deals. (EUROFER: THE EUROPEAN STEEL ASSOCIATON: , Situation of the European Steel Industry, 21 September 2020" [28.09.2020])

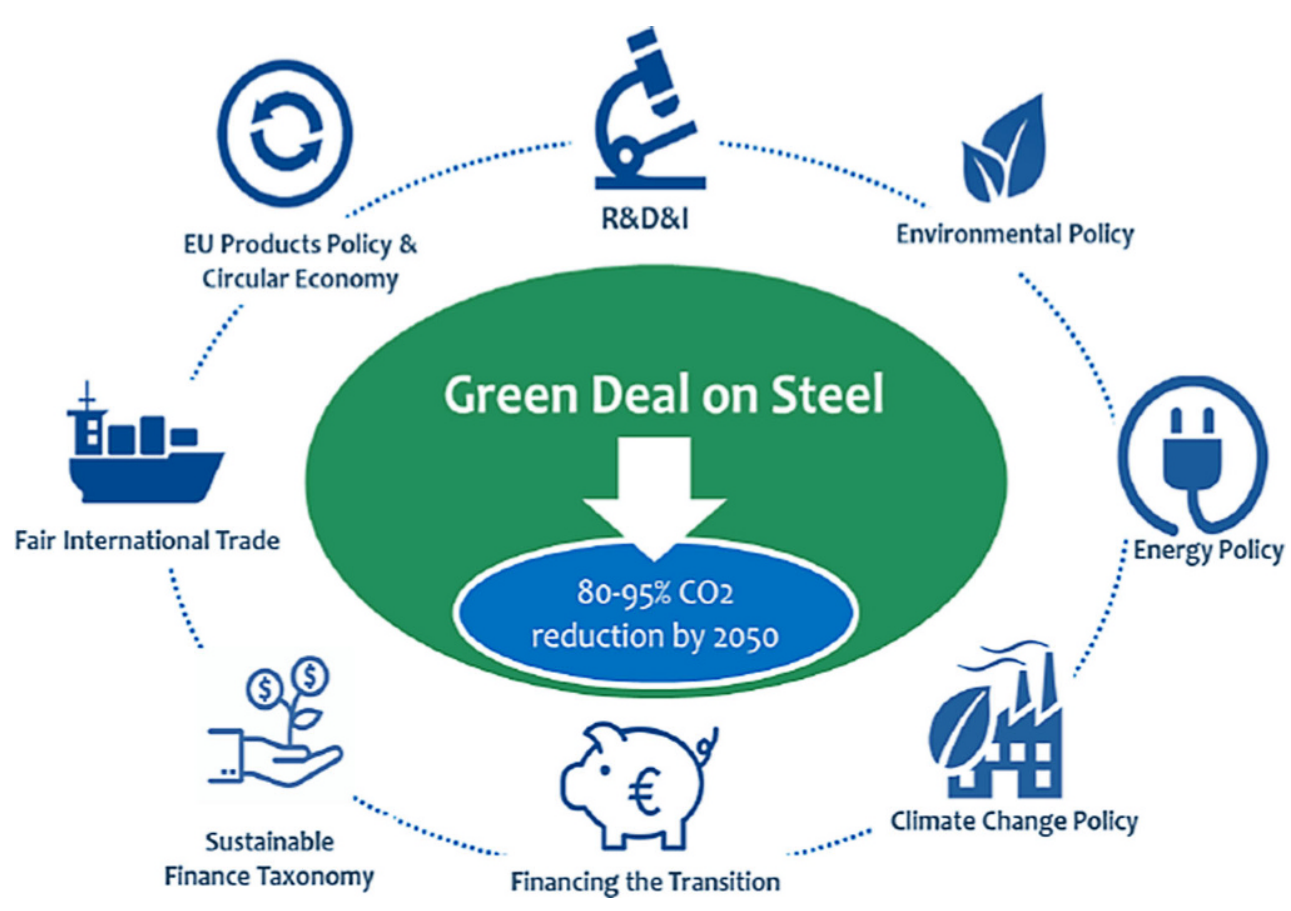

\section{Keine "grüne" Zukunft ohne mineralische Rohstoffe}

Die EU-Kommission hat im Green Deal ${ }^{15}$ die Schwerpunkte der neuen Amtsperiode auf Treibhausgasreduktion und Kreislaufwirtschaft gelegt und diese in der neuen Industriestrategie ${ }^{16}$ weiterentwickelt.

Break-Through-Technologien sollen künftig den Durchbruch für $\mathrm{CO}_{2}$-arme Produktion bringen, wie beispielsweise für die Produktion von "Green Steel." Für diese sind aber auch große Mengen nachhaltig erzeugter Energie nötig. Am Beispiel der E-Mobilität bedeutet dies einen massiven Mehrbedarf an mineralischen Rohstoffen sowohl für Batterien als auch für Elektromotoren und Ladestationen (s. Abb. 7 und 8).

Ähnlich verhält es sich mit der Herstellung erneuerbarer Energien durch Windturbinen, Solarzellen und geothermische Kraftwerke.

Die EU-Kommission stützt ihre Überlegungen im Rahmen des Green Deal und Kreislaufwirtschaftsaktionsplans ${ }^{17}$ unter anderem auf die Steigerung des Recyclings. Es wird jedoch immer mineralische Rohstoffe geben, die nicht recyclierbar sind, da sie entweder im Produktionsprozess verbraucht werden (z. B. Graphitelektroden in der Stahlindustrie) oder da sie weiterverarbeitet Teil eines Produktes sind (z. B. Eisenerz im Stahl). Andere mineralische Rohstoffe sind nicht oder kaum wirtschaftlich recyclierbar, da sie nur in sehr geringen Mengen in Produkten vorkom-

\footnotetext{
15 COM (2019)640 final: https://eur-lex.europa.eu/legal-content/DE/TXT/ PDF/?uri=CELEX:52019DC0640\&from=EN [14.09.2020]

16 COM (2020)102 final: https://eur-lex.europa.eu/legal-content/DE/TXT/ PDF/?uri=CELEX:52020DC0102\&from=DE [14.09.2020].

17 Circular Economy Action Plan: https://ec.europa.eu/environment/ circular-economy/index_en.htm [15.09.2020].
}

men (z.B. Seltene Erden) und Materialverbunde immer komplexer werden.

Darüber hinaus fordert auch der Green Deal und der Kreislaufwirtschaftsaktionsplan der EU-Kommission eine Verlängerung der Nutzungsdauer von Produkten, womit die Rohstoffe für einen noch längeren Zeitraum nicht in die Kreislaufwirtschaft zurückgeführt werden können (z.B. Aluminium, Kupfer, Stahl, Bauprodukte). Genau diese mineralischen Rohstoffe sind aber essentiell für "grüne" Materialien, Produkte und Technologien der Zukunft. Es wird folglich auch in Zukunft einen großen Bedarf an primären Rohstoffen geben, da auch langfristig durch Recycling nur ein Teil des Rohstoffbedarfs gedeckt werden kann (s. Abb. 9).

Gleichzeitig wird die Weltbevölkerung noch einige Jahrzehnte weiter steigen ${ }^{18}$, die Entwicklungsländer wollen verständlicherweise technologisch und sozial aufholen und auch der Protektionismus der rohstoffreichen Länder wird weiter zunehmen. Die Resilienz der Rohstoffbeschaffung nimmt weiter ab, wie eben erst die Corona-Pandemie offenbart hat.

Daher ist es essentiell darauf hinzuweisen, dass auch zukünftig, neben einer Steigerung des Recyclings, für eine funktionierende Kreislaufwirtschaft immer auch primäre mineralische Rohstoffe und folglich Bergbau in Österreich und Europa benötigt werden (s. Abb. 10). Die Fachverbände Bergbau-Stahl und NE-Metallindustrie unterstützten die Initiative der European Raw Materials Alliance (ERMA), deren Aktionsplan sich mit aktuellen und zukünftigen Herausforderungen auseinandersetzt und Innovationen fördert, um u. a. die Rohstoff-Abhängigkeit Europas gegenüber Drittländern zu reduzieren (s. Abschn. 5).

18 https://www.un.org/en/sections/issues-depth/population/index.html [14.09.2020]. 


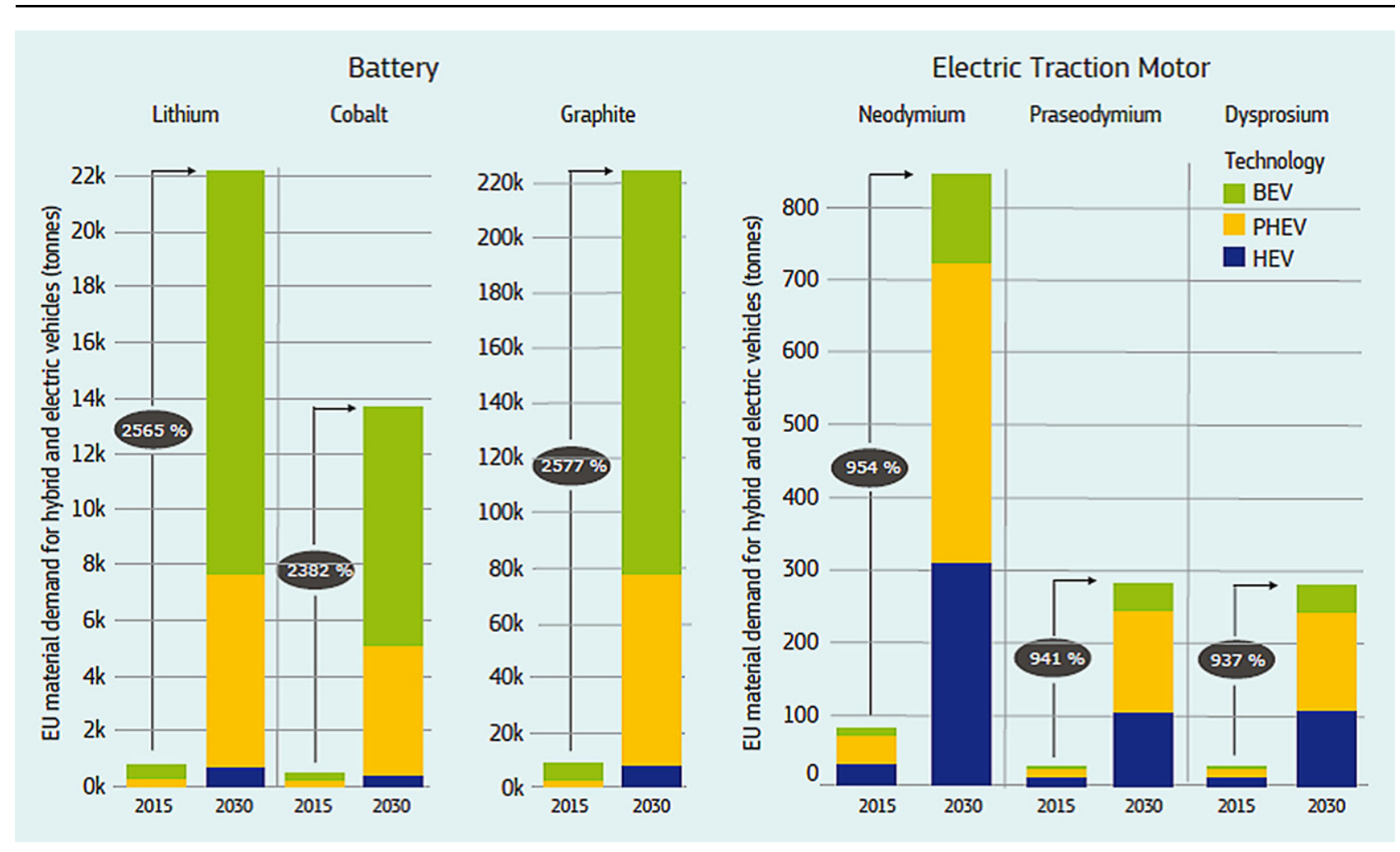

Abb. 7: Bedarf an mineralischen Rohstoffen für bestimmte Anwendungen. (European Commission, EIP on Raw Materials, Raw Materials Scoreboard 2018: https://doi.org/https://op.europa.eu/en/publication-detail/-/publication/117c8d9b-e3d3-11e8-b690-01aa75ed71a1 [14.09.2020])

\section{Liste kritischer Rohstoffe 2020}

Zur Unterstützung der Versorgungssicherheit mit mineralischen Rohstoffen erstellt die EU-Kommission seit 2011 eine Liste kritischer Rohstoffe (d.h. jene mit hohem Versorgungsrisiko und großer wirtschaftlicher Bedeutung), welche alle drei Jahre überarbeitet wird. Die Liste soll dazu beitragen, durch Förderung von Recyclingtätigkeiten einen Anreiz für die Erzeugung kritischer Rohstoffe in Europa zu schaffen und die Aufnahme neuer Abbautätigkeiten zu fördern. ${ }^{19}$ Es ist eine deutliche Steigerung der Anzahl der kritischen Rohstoffe - vor allem der Hochtechnologierohstoffe - erkennbar. Die Liste wurde im September 2020 um vier kritische Rohstoffe erweitert ${ }^{20}$ (s. Tab. 1).

\section{European Raw Materials Alliance (ERMA)}

Im Zuge der Veröffentlichung der überarbeiteten Liste wurde in der Mitteilung der EU-Kommission „Widerstandsfähigkeit der EU bei kritischen Rohstoffen: Einen Pfad hin zu größerer Sicherheit und Nachhaltigkeit abstecken “21 neben einem Aktionsplan mit zehn Maßnahmen für kritische

19 COM (2017) 490 final: https://ec.europa.eu/transparency/regdoc/rep/ 1/2017/DE/COM-2017-490-F1-DE-MAIN-PART-1.PDF [30.09.2020].

20 COM (2020) 474 final: https://eur-lex.europa.eu/legal-content/DE/TXT/ PDF/?uri=CELEX:52020DC0474\&from=EN [14.09.2020].

21 COM (2020) 474 final: https://eur-lex.europa.eu/legal-content/DE/TXT/ PDF/?uri=CELEX:52020DC0474\&from=EN [14.09.2020].
Rohstoffe ${ }^{22}$ auch eine neue Europäische Rohstoffallianz ${ }^{23}$ angekündigt. Damit soll als erster Schwerpunkt die Widerstandsfähigkeit der EU in der Wertschöpfungskette für Seltenen Erden und Magneten gestärkt werden. Zugang sollen alle relevanten Stakeholder haben - wie etwa die Industrie entlang der Wertschöpfungskette, Mitgliedstaaten, Zivilgesellschaft, Forschungsorganisationen, Investoren, Verbände und NGOs. Die am 29.09.2020 ins Leben gerufene ERMA hat sich zum Ziel gesetzt, Hindernisse zu beseitigen, um Chancen und Investitionen für die Versorgung mit mineralischen Rohstoffen sicherzustellen. Die EU-Kommission definiert das Ziel wie folgt: „Die Europäische Rohstoffallianz ist ein wichtiger Schritt zur Stärkung industrieller Ökosysteme, die von Rohstoffen abhängig sind. Sie wird die grünen und digitalen Übergänge beschleunigen, indem sie die Wertschöpfungsketten stärkt, das Angebot diversifiziert [... ].24 Das in der Industriestrategie ${ }^{25}$ angekündigte Bündnis ist die erste Maßnahme des Aktionsplans zu kritischen Rohstoffen ${ }^{26}$.

22 COM (2020) 474 final: https://eur-lex.europa.eu/legal-content/EN/ TXT/?uri=CELEX:52020DC0474[30.09.2020]

23 https://ec.europa.eu/germany/news/20200929-rohstoffallianz_de [30.09.2020].

24 https://erma.eu/eit-rawmaterials-will-manage-newly-launchedeuropean-raw-materials-alliance-erma/[30.09.2020].

25 https://ec.europa.eu/commission/presscorner/detail/de/ip_20_416 [30.09.2020].

26 https://ec.europa.eu/commission/presscorner/detail/de/ip_20_1542 [30.09.2020]. 
Abb. 8: Steigender Bedarf an mineralischen Rohstoffen für Erzeugung erneuerbarer Energien. (Arrobas, Daniele La Porta; Hund, Kirsten Lori; McCormick, Michael Stephen Ningthoujam, Jagabanta; Drexhage, John Richard. 2017 The Growing Role of Minerals and Metals for a Low Carbon Future (English). Washington, D.C.: World Bank Group https:// doi.org/http://documents. worldbank.org/curated/en/ 207371500386458722/TheGrowing-Role-of-Mineralsand-Metals-for-a-LowCarbon-Future [15.09.2020])

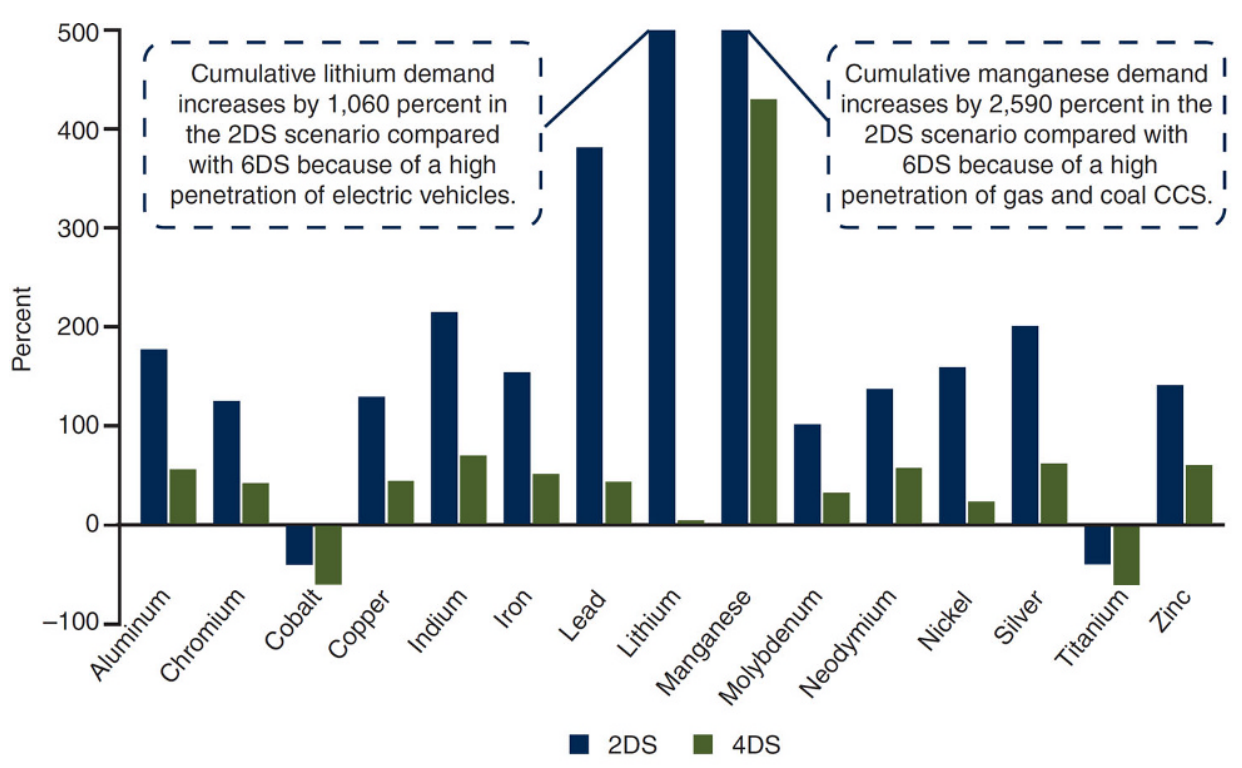

Note: $2 \mathrm{DS}=2$ degree scenario; $4 \mathrm{DS}=4$ degree scenario; $6 \mathrm{DS}=6$ degree scenario; $\mathrm{CSS}=$ carbon capture and storage

EOL-RIR 2020

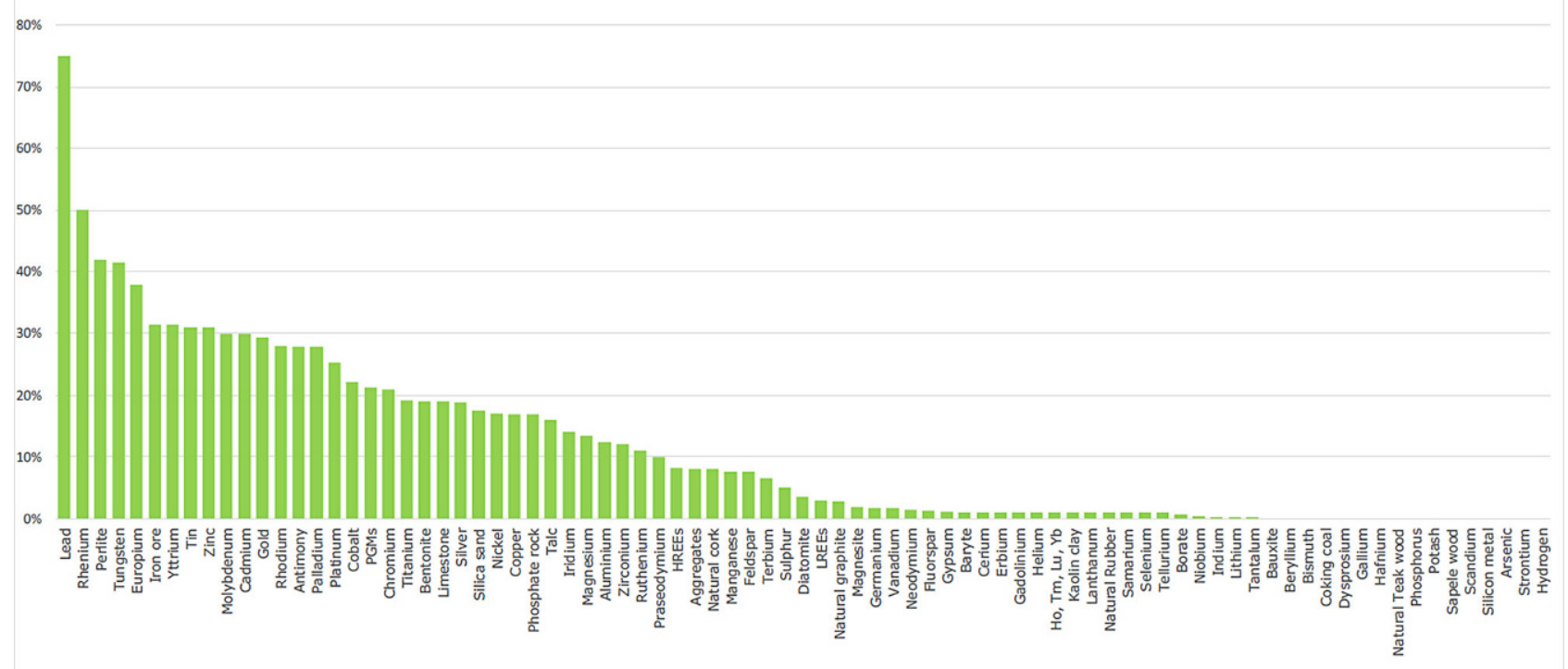

Abb. 9: Beitrag des Recyclings zur Deckung der Rohstoffnachfrage. (Study on the EU's list of Critical Raw Materials (2020) - Final Report: https://doi.org/ https://ec.europa.eu/docsroom/documents/42883/attachments/1/translations/en/renditions/native [14.09.2020])

\section{Carbon Border Adjustment Mechanism - wie realistisch?}

Vor dem Hintergrund des European Green Deals und der europäischen Bemühungen um einen weltweiten Klimaschutz steht das europäische Vorhaben eines $\mathrm{CO}_{2}$ Grenzausgleichsmechanismus (Carbon Border Adjustment Mechanism, CBAM). Ziel ist es zu verhindern, dass die Europäische Industrie, die für Klimaschutz und $\mathrm{CO}_{2}$-Reduktion in Europa höhere Kosten als die Konkurrenz aus dem EU-Ausland zu tragen hat, aus Europa abwandert. Dabei soll der Preis von Einfuhren in die EU den Emissionsgehalt und die damit im Exportstaat verbundenen Kosten widerspiegeln.
Die EU würde damit quasi die $\mathrm{CO}_{2}$-Emissionen anderer Staaten besteuern, wenn die entsprechenden Produkte in der EU konsumiert bzw. verarbeitet werden. Das mit dem Vorhaben verfolgte Ziel ist nachvollziehbar. Allerdings zeigen erste Dokumente der Europäischen Kommission, dass dieses schwer umsetzbar ist. Die Vorschläge reichen von einer Grenzsteuer für Importe, über eine Verbrauchersteuer bis zur Ausweitung des EU-ETS-Regimes auf EUImporte. Alle diese Varianten überzeugen bislang nicht, da viele Details offen sind. Gerade von diesen hängt aber der Erfolg dieser Maßnahme letztlich ab. Zudem muss ein $\mathrm{CO}_{2}$-Grenzsteuerausgleichsmechanismus einer Prüfung 
Abb. 10: EIT RawMaterials - Kreislaufwirtschaft. (EIT RawMaterials, https://doi. org/https://eitrawmaterials. eu/innovation-themes/ 03_06_ce-draft-1/[15.09.2020])

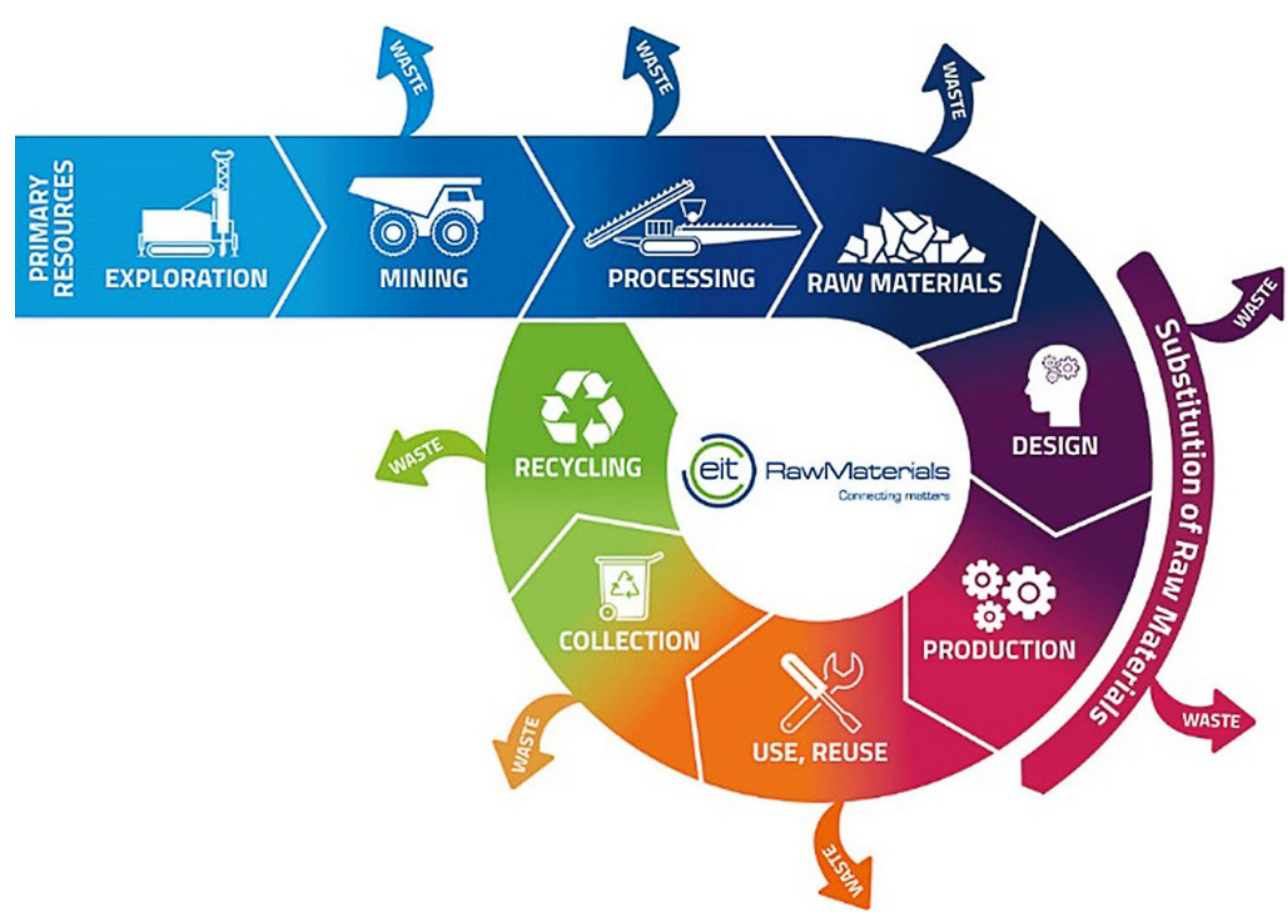

auf WTO-Konformität standhalten. Dies stellt eine große Herausforderung dar.

Was es jedenfalls zu verhindern gilt, sind Retorsionsmaßnahmen anderer Wirtschaftspartner/Wirtschaftsmächte, die Europa mit dieser Maßnahme einen „Protektionismus" vorwerfen könnten. Handelskriege gilt es unbedingt zu vermeiden, da sich diese auf die Rohstoffbeschaffung negativ auswirken würden. Und gerade in Bezug auf Rohstoffe ist Europa nach wie vor in hohem Maße importabhängig. Unabhängig von den handelsrechtlichen Implikationen, die ein $\mathrm{CO}_{2}$-Grenzausgleichsmechanismus auslösen könnte, würde eine Grenzsteuer, falls sie für inländische und ausländische Waren gleichermaßen eingeführt wird, den Import von Rohstoffen in die EU verteuern. Da die Folgen eines $\mathrm{CO}_{2}$-Grenzausgleichsmechanismus mannigfaltig und weitreichend sind, fordern die Fachverbände BergbauStahl und NE-Metallindustrie als Interessensvertretung die Durchführung einer umfassenden Wirkungsfolgenabschätzung vor Einführung einer derartigen Maßnahme.

\section{Green Finance und Taxonomy}

Der Mangel an Risikokapital für Bergbauprojekte und deren künftige Finanzierung in Österreich und im europäischen Raum stehen im engen Zusammenhang mit den derzeitigen Diskussionen zum Thema "sustainable finance" auf EU-Ebene. Die Europäische Union arbeitet derzeit an neuen Vorschriften zur Unterstützung ökologisch nachhaltiger Investitionen. Ziel ist es, Kapitalflüsse in nachhaltige Investitionen zu lenken. Dahinter stehen die Bemühungen Europas, die klima- und energiepolitischen Ziele umzusetzen, die im Rahmen des Pariser Klimaschutzübereinkommens und der Agenda 2030 der Vereinten Nationen für nachhaltige Entwicklung vereinbart wurden. Derzeit verwenden die
Anbieter von Finanzdienstleistungen eine Vielzahl unterschiedlicher Methoden, um die Nachhaltigkeit von Finanzprodukten zu bewerten. Das erschwert es Privatanlegern und kleinen institutionellen Anlegern, die Nachhaltigkeit einer Investition abzuschätzen. Einheitliche Kriterien sollen dazu führen, dass Anleger ausführlichere Informationen zur Nachhaltigkeit ihrer künftigen Investitionen erhalten, um eine bessere Vergleichbarkeit zu ermöglichen. Die Rohstoffgewinnung in der Europäischen Union ist strengen umweltrechtlichen Rahmenbedingungen unterworfen und als nachhaltig zu bezeichnen.

Daher setzen sich die Fachverbände Bergbau-Stahl und NE-Metallindustrie als Interessensvertretung dafür ein, die Definition von "sustainable finance“ derartig zu fassen, dass Aktivitäten der europäischen Rohstoffindustrie als nachhaltig im Sinne der künftigen EU-Taxonomie anerkannt werden. Dies ist wichtig, damit die Rohstoffbranche in Zukunft nicht von Finanzierungsquellen abgeschnitten wird. Investitionen in nachhaltige Rohstoffprojekte müssen attraktiviert werden.

\section{Chemikalienpolitik}

Im Kontext des European Green Deal beabsichtigt die Europäische Kommission noch im Jahr 2020 eine Chemikalienstrategie für Nachhaltigkeit ( „Chemical Strategy for Sustainability“) zu veröffentlichen. Die neue Chemikalienstrategie soll auf den Ergebnissen der letzten REACHEvaluierungen und diverser Fitness-Checks aufbauen. Dabei sollen Schnittstellen der EU-Chemikaliengesetzgebung zu anderen Rechtsbereichen, insbesondere Produkt- und Abfallrecht, aber auch zum Lebensmittel- und Arbeitnehmerschutz stärker beachtet werden. Es gilt, eine Reihe von Schwachstellen, Überschneidungen oder Widersprüchen 


\begin{tabular}{|lll}
\hline $\begin{array}{l}\text { TABELLE 1 } \\
\text { Liste kritischer Rohstoffe der EU-Kommission (30) (Update 2020). (COM (2020) 474 final https://doi.org/https:// } \\
\text { eur-lex.europa.eu/legal-content/EN/TXT/PDF/?uri=CELEX:52020DC04748from=EN [14.09.2020]) }\end{array}$ \\
\hline Antimon & Germanium & Metalle der Platingruppe \\
\hline Baryt & Hafnium & Phosphorit \\
\hline Bauxit & Schwere Seltene Erden & Phosphorus \\
\hline Beryllium & Leichte Seltene Erden & Scandium \\
\hline Wismuth & Indium & Siliciummetall \\
\hline Borat & Lithium & Strontium \\
\hline Kobalt & Magnesium & Tantal \\
\hline Kokskohle & Natürlicher Graphit & Titan \\
\hline Flussspat & Naturkautschuk & Vanadium \\
\hline Gallium & Niob & Wolfram \\
\hline
\end{tabular}

zu adressieren. Wesentlich wird dabei aus Sicht der Industrie sein, dass es in Zukunft klare Zuständigkeiten, Arbeitsund Ressourcenverteilung zwischen den unterschiedlichen EU-Agenturen sowie diversen wissenschaftlichen Ausschüssen bzw. den Kommissionsdienststellen und den mitgliedstaatlichen Behörden gibt.

Die neue Strategie will dazu beitragen, EU-Bürger und die Umwelt besser vor gefährlichen Chemikalien zu schützen, aber auch Innovationen für ungefährliche und nachhaltige Chemikalien voranzutreiben. Der Ansatz „ein Stoff - eine Bewertung" soll in Zukunft gestärkt werden. Es soll mehr Transparenz geben, wenn bestimmte Maßnahmen in Bezug auf Chemikalien priorisiert werden. Außerdem will man wissenschaftliche Fortschritte in den Regularien neu abbilden; dies betrifft Risiken, die mit endokrinen Disruptoren verbunden sind, in Produkten enthaltene gefährliche Chemikalien einschließlich Importprodukten, Kombinationseffekte, die von verschiedenen Chemikalien ausgehen und sehr persistente Chemikalien.

Die neue Chemikalienstrategie ist für die Bergbau- und Metallbranchen von großer Bedeutung. Gerade Metalle haben oft gefährliche Eigenschaften, schaffen aber im Falle einer sicheren Verwendung einen hohen sozioökonomischen Nutzen für die Gesellschaft. Beispielsweise sind Metalle mit karzinogenen Eigenschaften in Batterien oder Solarpanelen enthalten; diese sind jedoch für die Verwirklichung des European Green Deal und den Übergang zu einer klimaneutralen Produktion unerlässlich.

Die Fachverbände Bergbau-Stahl und NE-Metallindustrie empfehlen, einen stärkeren Fokus auf Beherrschung von Exposition und Risikomanagement/Risikokontrolle anstatt jenen auf Gefahren bzw. gefährliche Eigenschaften. Ziel sollte es sein, sicherzustellen, dass von gefährlichen Stoffen ausgehende Risiken während des gesamten Lebenszyklus eines Produktes ausreichend beherrscht werden können.

\section{Zusammenfassung}

Zusammenfassend ist die österreichische Mineralrohstoffwirtschaft auf eine kohärente Rohstoff- und Energiepolitik in Europa angewiesen. Maßnahmen müssen stringent im Rahmen des Green Deals, der Industriestrategie und der European Raw Materials Alliance (ERMA) entwickelt und umgesetzt werden. Gleichzeitig muss ein Carbon-Leakage-Szenario unbedingt vermieden werden. Europäische Vorhaben wie der $\mathrm{CO}_{2}$-Grenzausgleichsmechanismus sind nicht ausreichende Versuche, die Abwanderung der Europäischen Industrie zu verhindern. Zusätzlich ist es wichtig, Abhängigkeiten von asiatischen Lieferanten zu verringern, und gleichzeitig die europäischen Wertschöpfungsketten umfassend zu stärken. Dies ist insbesondere in der durch die Pandemie verursachte Wirtschaftskrise essentiell. Die Aufbau- und Resilienzfinanzhilfen müssen zielgerichtet die im internationalen Wettbewerb stehende Mineralrohstoffindustrie unterstützen.

Die Transformation zu einer nachhaltigen Wirtschaft und Gesellschaft erfordert einen großen Mehrbedarf an mineralischen Rohstoffen und elektrischer Energie. Die darüber hinaus gehende Forderung der EU-Kommission (Green Deal, Kreislaufwirtschaftsplan) einer Verlängerung der Nutzungsdauer von Produkten bewirkt, dass Rohstoffe über längere Zeit der Kreislaufwirtschaft zurückgeführt werden können (z. B. Aluminium, Kupfer, Stahl, Bauprodukte). Genau diese sind aber essentiell für "grüne" Materialien, Produkte und Technologien der Zukunft. Es wird folglich auch in Zukunft einen großen Bedarf an primären Rohstoffen geben, da auch langfristig durch Recycling nur ein Teil des Rohstoffbedarfs gedeckt werden kann. Nur durch eine Stärkung der Wertschöpfungsketten kann eine ausreichende Versorgung gewährleistet werden

Das Thema Nachhaltigkeit wird auch für Investoren immer wichtiger (sustainable finance). Österreich und der europäischen Raum verzeichnen einen Mangel an Risikokapital für Bergbauprojekte und deren künftige Finanzierung. Unterschiedliche Methoden zur Bewertung der Nachhaltigkeit eines Finanzproduktes erschweren es Privatanlegern und kleinen institutionellen Anlegern, eine genaue Abschätzung zu treffen. Einheitliche Kriterien sollen dazu führen, dass Anleger ausführlichere Informationen zur Nachhaltigkeit ihrer künftigen Investitionen erhalten. Die Fachverbände Bergbau-Stahl und NE-Metallindustrie setzen sich als Interessensvertretung dafür ein, die Definition von "sustainable finance“ derartig zu fassen, dass Aktivitäten der europäischen Rohstoffindustrie als nachhaltig im Sinne der künftigen EU-Taxonomie anerkannt werden. 